\title{
Oral Insulin Delivery by Chitosan Coated Solid Lipid Nanoparticles: Ex vivo and in vivo Studies
}

\author{
Rakshitha Bhaskar Anchan, Marina Koland* \\ Department of Pharmaceutics, NGSM Institute of Pharmaceutical Sciences (NGSMIPS), NITTE (Deemed to be University), Deralakatte, Mangalore, Karnataka, INDIA.
}

\begin{abstract}
Objectives: The objective of this investigation was to formulate chitosan coated, insulin-loaded solid lipid nanoparticles (SLN) for oral administration and investigate their potential as an effective alternative to the subcutaneous injection. Methods: The SLN were prepared from glyceryl monostearate and coated with the mucoadhesive polymer, chitosan and were investigated for physical properties, ex vivo permeation through Caco-2-cell monolayer and goat intestinal mucosa. The in vivo efficacy of the optimized formulation in controlling blood glucose levels was studied in streptozotocin induced diabetic rats. Results: Coating in the presence of Poloxamer 407 resulted in significantly smaller nanoparticles than those with Tween 80. Scanning electron microscopy and transmission electron microscopy revealed spherical particles of uniform size distribution. The insulin association efficiency and loading efficiency of SLN prepared with Poloxamer were much greater than those with Tween 80. Ex vivo permeability studies in Caco-2 cell monolayer revealed a 4 fold increase in insulin permeation from chitosan coated SLN $(P<0.005)$ as compared to uncoated SLN at the end of $6 \mathrm{~h}$. The percentage permeation through excised sheep intestinal mucosa from coated SLN was twice that
\end{abstract}

from uncoated SLN and nearly 20 times from insulin solution. The oral administration of chitosan-coated insulin SLN to streptozotocin-induced diabetic rats resulted in a significant hypoglycemic effect $(P<0.05)$ when compared to the groups that received uncoated insulin-loaded SLN or the oral insulin solution and was comparable to that of subcutaneous insulin at the end of an $8 \mathrm{~h}$ study. Conclusion: Chitosan coated SLN can be considered promising as an effective oral insulin formulation.

Key words: Chitosan, Diabetes, Glyceryl monostearate, Insulin, Nanoparticles, Permeation.

Correspondence

Dr. Marina Koland

NGSM Institute of Pharmaceutical Sciences, NITTE (Deemed to be University), Deralakatte, Mangalore-575018, Karnataka, INDIA.

Phone: +919886042035

Email: marinakol@nitte.edu.in

DOI: 10.5530/jyp.2021.13.10

\section{INTRODUCTION}

Patients suffering from Type I also known as Insulin Dependent Diabetes Mellitus (IDDM) or juvenile onset diabetes mellitus and in the later stage of Type II - Noninsulin Dependent Diabetes Mellitus (NIDDM) or maturity onset diabetes mellitus, require an exogenous supply of insulin for their survival. Insulin is used to maintain the blood glucose level in these diabetic patients. ${ }^{1}$ The most common mode of administration of insulin is by subcutaneous injection. However, this route is associated with several problems such as local discomfort, pain, allergic reactions, poor patient compliance and hypoglycemia due to overdose. To overcome these problem several approaches for the novel drug delivery of insulin were explored. ${ }^{2,3}$

The oral route is considered to be more convenient, safe and the most acceptable route of administration of drugs for chronic therapy. However insulin administered by oral route is not as effective as by subcutaneous route due to poor absorption in gastrointestinal tract and also due to its large molecular size and hydrophilic nature. As a protein, insulin is degraded by gastric acid or proteolytic enzymes present in GI tract which act as the most important barrier limiting the absorption of insulin. ${ }^{4}$ Therefore a lot of research has been carried out to make insulin available for the oral treatment of Diabetes. To develop an adequate oral delivery system, the maintenance of insulin biological stability in the gastrointestinal tract (GIT) and in the enterocytes must be taken into consideration during the formulation process. Several novel insulin delivery systems, such as intestinal patches, hydrogels, microparticles and nanoparticles, have been explored to deliver insulin by paracellular and/or transcellular transport throughout the ileum and colon. ${ }^{5}$ The delivery systems used may contain excipients, which protect insulin from aggregation and enzymatic degradation, prolong its residence time in the GIT and enhance its intestinal uptake. Various polymeric nanoparticles, liposomes and a lipid-based system where developed to increase the bioavailability of insulin through oral administration. It was reported that nanoparticles of biodegradable polymer show better control over the oral delivery of proteins and peptides. The main advantage of nanoparticles is that they can pass through the gastrointestinal (GI) tract and which is taken up by $M$ cells of Payer's patches, a type of lymphatic island in the intestine which is the main site through which nanoparticles get absorbed when compared to microparticles. ${ }^{6}$ In fact studies have shown that nanoparticles can temporarily and reversibly open the tight junctions in Caco-2 cell monolayers, thus increasing their paracellular permeability. ${ }^{7}$ However, the properties of the carrier can strongly influence its uptake.

Solid lipid nanoparticles (SLN) are submicron colloidal carriers (50$1,000 \mathrm{~nm}$ ) made from lipids which are highly purified triacylglycerols, complex acylglycerol mixtures or waxes and dispersed either in water or in an aqueous surfactant solution. ${ }^{8}$ SLN show several advantages over other drug carriers such as good tolerability biodegradation and the possibility of large industrial scale production. ${ }^{9,10}$ Studies have reported that nanoencapsulation of proteins in lipid nanoparticles can improve their bioavailability, prolong their blood residence time and/or modify their biodistribution. ${ }^{11,12}$ The use of chitosan, a natural cationic polysaccharide derived from chitin by partially deacetylating its acetamide groups, for the coating of nanoparticles may be able to increase the penetration of

This is an open access article distributed under the terms of the Creative Commons Attribution-NonCommercial-ShareAlike 4.0 License, which allows others to remix, tweak, and build upon the work non-commercially, as long as the author is credited and the new creations are licensed under the identical terms. 
the encapsulated macromolecules in mucosal surfaces. Chitosan has the advantages of low toxicity, good biocompatibility, antimicrobial and absorption enhancing properties. ${ }^{13}$ Moreover, chitosan being a mucoadhesive polmer, is able to localize the particles to the absorption sites, increasing the possibility of better absorption. It has also been reported that the absorption-promoting effect of chitosan is due to the improved mucoadhesion and temporary opening of tight junctions in the mucosal cell membrane which is responsible for the targeting of the drug and uptake by the Peyer's patches. ${ }^{14,15}$

Thus the purpose of this work was to explore the possibility of developing insulin loaded SLN intended for oral administration and coated with the mucoadhesive polymer, chitosan for localizing the particles to the mucosal surface and enhancing their uptake.

\section{MATERIALS AND METHODS}

\section{Materials}

Human lyophilized insulin (100 mg per vial) was procured from Sigma Aldrich, Mumbai. The SLN were prepared from lipids such as Glycerol monostearate (GMS). Chitosan (95\% deacetylated) and GMS were procured from Lobo Chemie Pvt. Ltd, Mumbai. Poloxamer 407 and Tween 80 were purchased from Yarrow Chem Products Pvt. Ltd., Mumbai. All other chemicals were of analytical grade and obtained from Lobo Chemie Pvt Ltd., Mumbai.

\section{Methods}

\section{Preparation of SLN of insulin}

The SLN were prepared by the modified solvent emulsificationevaporation method using w/o/w double emulsion technique. Briefly, the lipid was dissolved in the organic solvent in a glass test tube in a bath sonicator to form the oil phase. To avoid loss of solvent by evaporation during handling, closed tubes were used. The insulin was dissolved in hydrochloric acid solution $(0.1 \mathrm{M})$ by slight shaking to form an internal water phase. The internal water phase was added to an oil phase and sonicated in an ice bath at 70\% amplitude for $30 \mathrm{sec}$ to form a w/o emulsion. The emulsion formed was then poured into a beaker containing external water phase with surfactant and sonicated at $70 \%$ amplitude for $30 \mathrm{sec}$. A w/o/w emulsion was obtained which was stirred on a magnetic stirrer at $300 \mathrm{rpm}$ for the evaporation of the organic solvent, producing an SLN dispersion. The compositions of the aqueous and oil phase used for the formulations is shown in Table 1.

The SLN formulation was then coated with chitosan. Briefly, the SLN dispersion was poured into a beaker containing $0.5 \% \mathrm{w} / \mathrm{v}$ chitosan and $2 \% \mathrm{w} / \mathrm{v}$ surfactant (Poloxamer 407 or Tween 80 ) in 1\% w/v acetic acid. The dispersion was stirred overnight and chitosan coated SLN were formed. ${ }^{16}$

Table 1: Composition of SLN formulations.

\begin{tabular}{|c|c|c|c|}
\hline $\begin{array}{l}\text { Product } \\
\text { code }\end{array}$ & $\begin{array}{c}\text { Internal aqueous } \\
\text { phase }\end{array}$ & Oil (lipid) phase* & $\begin{array}{c}\text { External aqueous } \\
\text { phase }\end{array}$ \\
\hline $\mathrm{F} 1$ & $\begin{array}{c}\text { Insulin }(1 \mathrm{mg}) \\
\text { in } 0.2 \\
\mathrm{ml} \text { of } 0.1 \mathrm{M} \mathrm{HCl}\end{array}$ & $\begin{array}{c}\text { GMS }(250 \mathrm{mg}) \\
\text { in chloroform } \\
(2 \mathrm{ml})\end{array}$ & $\begin{array}{c}\text { Poloxamer } 407 \\
(2 \% \mathrm{w} / \mathrm{v} \\
20 \mathrm{ml})\end{array}$ \\
\hline F2 & $\begin{array}{c}\text { Insulin }(1 \mathrm{mg}) \\
\text { in } 0.2 \\
\mathrm{ml} \text { of } 0.1 \mathrm{M} \mathrm{HCl}\end{array}$ & $\begin{array}{l}\text { GMS }(250 \mathrm{mg}) \text { in } \\
\text { chloroform }(2 \mathrm{ml})\end{array}$ & $\begin{array}{c}\text { Tween } 80(2 \% \mathrm{w} / \mathrm{v} ; \\
20 \mathrm{ml})\end{array}$ \\
\hline
\end{tabular}

${ }^{\star}$ GMS - glyceryl monostearate

\section{Evaluation of Chitosan coated SLN of insulin}

\section{Particle size and size distribution}

The particle size and polydispersity index of SLN's was determined by dynamic light scattering using Zeta sizer (Nano ZS Malvern, UK).

\section{Zeta potential measurement}

The zeta potential of SLN's was measured by using the zeta sizer (Nano ZS Malvern, UK) which uses a combination of Laser Dropper velocimetry (DLV) and Phase Analysis Light Scattering (PALS) by a patented technique called M-3 PALS to measure the particle electrophoretic mobility.

\section{Morphological analysis of coated SLN by Scanning Electron Microscopy} (SEM)

The surface morphology of solid lipid nanoparticles was determined by SEM (Zeiss Sigma 300). Before analysis, samples were freeze dried and a small amount was placed on a metal stub and subjected to gold sputtering. These coated samples were then scanned and photomicrographs were taken at an acceleration voltage of $15 \mathrm{kV}$.

\section{Transmission Electron Microscopy (TEM) of coated SLN}

Particle size analysis was also carried out by TEM (Jeol/JEM 2100 High Resolution). A drop of the diluted selected chitosan coated SLN formulation was placed on carbon-coated copper grids and dried under a lamp before the microscopic images were recorded.

\section{Insulin association efficiency}

The Association Efficiency (AE) was determined by the indirect method as reported by Fonte et al. ${ }^{17}$ The amount of insulin entrapped into SLN was calculated by the difference between the total amount used to prepare the SLN and the amount of insulin that remained in the aqueous phase. The aqueous phase was separated by centrifugation and filtration. The sample was centrifuged at $4000 \mathrm{rpm}$ for $1 \mathrm{hr}$. The separated supernatant was diluted and passed through a $0.2 \mu \mathrm{m}$ syringe filter. The insulin concentration in the filtrate was determined by HPLC-UV method. The equation used to determine $\mathrm{AE}$ is given as follows:

A.E. $=\frac{\text { Total amount of insulin }- \text { Free insulin in filtrate }}{\text { Total amount of insulin }} \times 100$

The loading efficiency (LE) was determined from the difference between the total amount of insulin initially used to prepare the nanoparticles and the amount of residual un-associated or free insulin after particle separation as a percentage of total nanoparticle dry mass. As described above for $\mathrm{AE}$, the free insulin present in the filtrate after separation of the SLN was measured by HPLC-UV. The total dry weight of the nanoparticles was determined. The residue remaining after centrifugation was combined with the rinsing from the syringe filter and the dispersion so obtained was subjected to freeze drying. The LE was then calculated using the formula below. ${ }^{17}$

L.E. $=\frac{\text { Total amount of insulin }- \text { Free insulin in filtrate }}{\text { Total weight of nanoparticles }} \times 100$

Ex vivo permeability studies using Caco-2 cell monolayer model

The potential of the insulin loaded chitosan coated SLN to permeate through the gastro-intestinal barrier was assessed ex vivo by using the Caco- 2 cell monolayer as the model membrane.

Caco- 2 cells were cultured at $37^{\circ} \mathrm{C}$ in an atmosphere of $5 \% \mathrm{CO}_{2} / 95 \%$ air and 90\% relative humidity in Dulbecco's modified Eagle medium (DMEM) supplemented with $1 \%$ penicillin-streptomycin, $2 \mathrm{mM}$ L-glutamine, $1 \%$ nonessential amino acids and $10 \%$ heat-inactivated fetal bovine serum. ${ }^{18,19}$ 
The cultured cells were seeded on 24 well plates (Transwell) in DMEM medium at a density of 10,000 cells/ well. Transepithelial electrical resistance (TEER) measurements were carried out to monitor the growth and viability of the monolayer. The apical and basolateral chambers represent the luminal and blood side of the gastro-intestinal tract respectively. After 20 days from initial seeding, aliquots of $100 \mu \mathrm{l}$ each of insulin solution and SLN dispersions equivalent to $50 \mu \mathrm{g} / \mathrm{ml}$ are applied to the apical side of the cells..$^{20}$ At the end of $6 \mathrm{~h}$ the supernatant was separated and the cells were washed with phosphate buffered saline of $\mathrm{pH}$ 7.4. Then the cells were scraped out from wells and subjected to centrifugation at $1200 \mathrm{rpm}$ at $4^{\circ} \mathrm{C}$ for $5 \mathrm{~min}$. The cells were collected and the drug permeated into the cells was extracted with $0.1 \mathrm{M} \mathrm{HCl}$ and the amount of drug permeated was then analysed by HPLC-UV method. The data was analysed by Student's $t$ test using Statistical Package for the Social Sciences (SPSS) software (SPSS, Chicago, IL, USA) with $P$ values $<0.05$ indicating statistical significance.

\section{Ex vivo permeation studies using intestinal mucosa of goat}

For this study, the intestinal mucosa of a goat was obtained fresh from the local slaughter house. ${ }^{21}$ The goat intestinal mucosa was trimmed off to remove any extraneous tissue and rinsed with phosphate buffered saline ( $\mathrm{pH}$ 6.8) to remove mucous and lumen contents. The tissue was mounted between the donor and the receptor compartment of a Franz diffusion cell with the mucosal surface facing the donor compartment. Formulation equivalent to $50 \mu \mathrm{g} / \mathrm{ml}$ of insulin was placed in the donor compartment, while the receptor compartment was filled with $25 \mathrm{ml}$ of phosphate buffer $\mathrm{pH} 6.8$ and its temperature was maintained at $37^{\circ} \mathrm{C}$. The contents of the receptor compartment was stirred using a magnetic stirrer at $100 \mathrm{rpm}$. An aliquot of $2 \mathrm{ml}$ was withdrawn from the receptor compartment at suitable time intervals and replaced with the same volume of fresh medium. These samples were analyzed using HPLC-UV. The study was conducted for a period of $6 \mathrm{~h}$.

\section{In vivo anti-diabetic studies using albino rats}

The animal experiments were carried out in adherence to the CPCSEA guidelines and after obtaining permission from the Institution Animal Ethics Committee of the NGSM Institute of Pharmaceutical Sciences, Mangalore.

Male albino rats of body weight, 200-250 g were housed in controlled environmental conditions of temperature $\left(22 \pm 2^{\circ} \mathrm{C}\right)$ and relative humidity (45-65\%). The rats were fed with standard diet feed and were provided fresh water. Lighting was on standard $12 \mathrm{~h}$ on/12 h off cycle.

Total of six groups of animals was taken and treatment was given as follows:

Group 1: Normal control, are treated with distilled water.

Group 2: Negative control without any treatment.

Group 3: Positive control with subcutaneous insulin (2.5 IU/kg)

Group 4: Oral insulin solution (20 IU/kg, in Phosphate Buffered Saline). Group 5: Uncoated solid lipid nanoparticles (25 IU/kg)

Group 6: Chitosan coated solid lipid nanoparticles (25 IU/kg)

Diabetes was induced in Groups 2 - 6 as per the method described. A single intra-peritoneal injection of streptozotocin $(10 \mathrm{mg} / \mathrm{ml}$ in citrate buffer, $\mathrm{pH} 4.5$ ) at a concentration of $60 \mathrm{mg} / \mathrm{kg}$ of body weight was administered to each rat. After 3 days, rats presented fasted blood glucose levels above $250 \mathrm{mg} / \mathrm{dL}$. These animals were used and were fasted $12 \mathrm{~h}$ before the experiment and remained fasted during the experiment, but had free access to water.

Groups 3- 6 were administered the treatments as indicated above. Blood samples were collected from the tail vein after needle puncture at pre- determined time intervals from each animal. The samples were collected for a period of $8 \mathrm{~h}$ from all animal groups and the blood glucose level was measured using a glucometer. The results obtained were statistically evaluated by one-way analysis of variance (ANOVA). The significant differences $(p<0.05)$ between the groups were compared using post-hoc test (Scheffe). All statistical analysis was performed using the Statistical Package for the Social Sciences (SPSS) software. ${ }^{22-24}$ Plasma glucose levels were plotted as percentage of base line glucose levels against time.

\section{RESULTS}

Solid lipid nanoparticles were prepared from GMS as lipid and two surfactants, Poloxamer 407 and Tween 80 using solvent emulsificationevaporation method. The SLN were coated with chitosan to impart mucoadhesiveness and improve permeation of the nanoparticles through the intestinal mucosa. ${ }^{25} \mathrm{~F} 3$ and F4 are the coated forms of F1 and $\mathrm{F} 2$ respectively.

The results for particle size, zeta potential and PDI of all formulations are displayed in Table 2.

The SEM analysis indicated that the chitosan coated insulin solid lipid nanoparticles were spherical and separate. The photomicrograph of formulation F3 and F4 (Figure 1) shows that the former had surfaces that were smoother than those of the latter. F4 also appeared to consist of much larger particles probably due to increased chitosan deposition.

The TEM Images (Figure 2) of F1 and F3 confirm that the coated and uncoated SLN were spherical in shape and indicated a uniform size distribution. The average size of particles in the micrograph for F1 was found to be $220 \pm 3 \mathrm{~nm}$ and that of F3 was $403 \pm 2 \mathrm{~nm}$. The larger size of F3 was the result of the chitosan coating on the SLN surface.

The data for association efficiency (AE) and loading efficiency (LE) of all formulations is given in Table 2 .

Ex vivo permeation studies using Caco-2 cell monolayer model were carried out to assess the efficiency of the nanoparticles to permeate through intestinal epithelium. The results obtained for coated and uncoated formulations were compared in Figure 3.
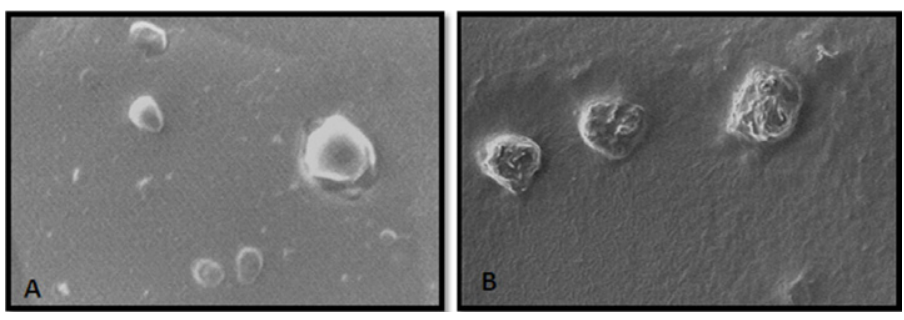

Figure 1: Scanning Electron Microscopic images of F3 (A) and F4 (B).

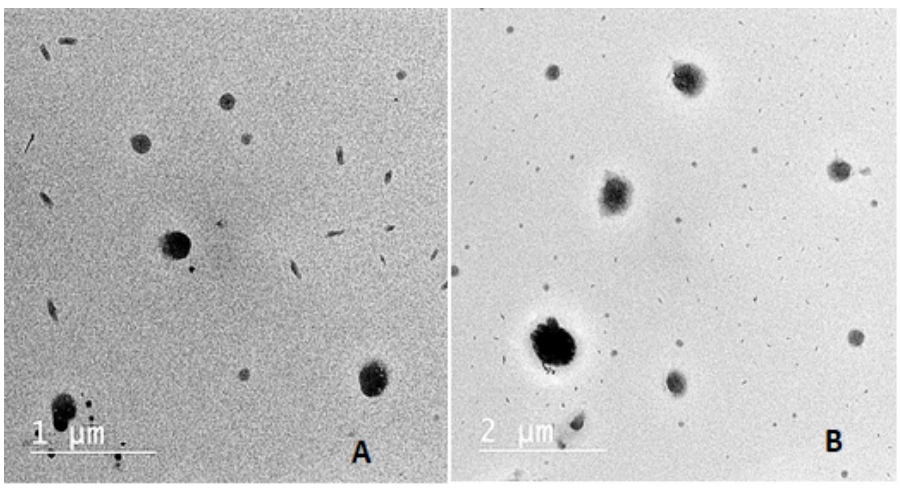

Figure 2: Transmission Electron Microscopic images of F1 (A) and F3 (B). 
The permeation profiles of insulin through intestinal mucosa of goat from coated and uncoated SLN are depicted in Figure 4. Both the coated and uncoated formulations provided a sustained release of insulin over the observed period of $6 \mathrm{~h}$. The in vivo antidiabetic efficacy of the SLN formulations were compared with that of subcutaneous insulin in streptozotocin induced diabetic male albino rats as described under 'Methods' and the minimum glucose concentration (\% initial) was determined. The blood glucose profiles in terms of percentage glycemia produced in the different groups of rats during the study are presented in Figure 5.

\section{DISCUSSION}

Ideally for nanoparticles, a particle size below $500 \mathrm{~nm}$ is necessary to enhance the absorption through intestinal mucosa. ${ }^{26-28}$ It is observed that, irrespective of the surfactant used, the particle size of the uncoated SLN (F1 and F2) were less than $400 \mathrm{~nm}$. Thus GMS was considered a suitable lipid for the preparation of SLN. Coating resulted in an increase in particle size and, the choice of the surfactant did make a difference to the final size. Coated SLN of formulation F3 using Poloxamer 407 as surfactant were significantly smaller than those of F4 with Tween 80 . This could be due to the increased deposition of chitosan on the SLN surface brought about by the former's decreased solubility in the presence of Tween 80 as compared to Poloxamer 407.

The lower value of zeta potential is normal for particles containing non-ionic surfactants like Tween 80 and Poloxamer 407. After chitosan coating, the surface of the particles would be positively charged due to

Table 2: Data for particle size, PDI, zeta potential, AE and LE for all formulations.

\begin{tabular}{cccccc}
\hline $\begin{array}{c}\text { Product } \\
\text { code }\end{array}$ & $\begin{array}{c}\text { Particle } \\
\text { size }(\mathrm{nm})\end{array}$ & PDI & $\begin{array}{c}\text { Zeta } \\
\text { potential } \\
(\mathrm{mV})\end{array}$ & AE (\%)* & LE (\%)* \\
\hline F 1 & 225.3 & 0.217 & -12.62 & $50.11 \pm 0.31$ & $19.25 \pm 1.56$ \\
F 2 & 315.7 & 0.255 & -6.18 & $31.52 \pm 0.12$ & $12.16 \pm 2.11$ \\
F 3 & 395.3 & 0.226 & 9.37 & $57.23 \pm 0.15$ & $20.53 \pm 1.25$ \\
F 4 & 784.2 & 0.201 & 6.82 & $33.41 \pm 0.12$ & $11.47 \pm 2.03$ \\
\hline
\end{tabular}

${ }^{*}$ Average of 3 measurements with standard deviation

AE - Association Efficiency; LE - Loading Efficiency

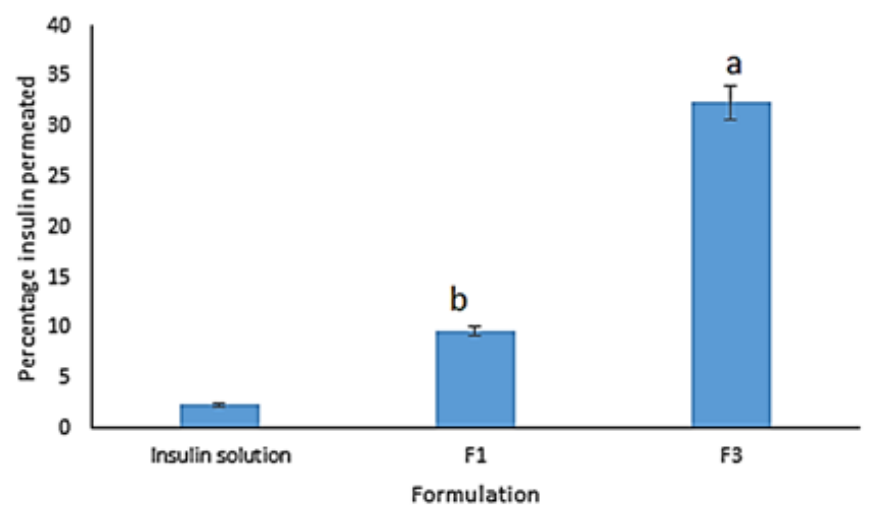

Figure 3: Comparison of insulin permeation through Caco-2 cell monolayer at the end of $6 \mathrm{~h}$ from SLN formulations F1 and F3 with Insulin solution.

All values are average of 3 determinations with standard deviation. $a-P<0.005$ when compared to insulin solution and $F 1, b-P<0.05$ when $F 1$ was compared to insulin solution the cationic nature of the polymer thus confirming its deposition of on the surface of the SLN. ${ }^{29,30}$ The values of zeta potential of all formulations are within the range of $\pm 25 \mathrm{meV}$ indicating that the nanoparticles were stable with less tendency to aggregate.

The PDI of all formulations were found to be less than 0.3 indicating a narrow size distribution.

The association efficiency reflects the fraction of the total insulin that is actually entrapped in the lipid. The composition of the lipids can influence both association efficiency and drug loading efficiency. Since hydrophilic drugs such as insulin tend to get expelled from the lipid matrix during the solidification of the SLN, drug loading can be difficult. ${ }^{31}$

The choice of the lipid and surfactant makes a difference to both $\mathrm{AE}$ and LE. Some of the factors that determine the drug loading capacity of SLNs are the solubility of drug in lipid phase and the chemical or physical structure of solid lipid matrix. ${ }^{32}$ GMS is a good emulsifier and a suitable combination with a surfactant such as Tween 80 or Poloxamer 407 can improve the AE and LE for hydrophilic drug, insulin by enhancing solubilization in the lipid matrix. It was also observed that when Poloxamer 407 was used as the surfactant, there was a substantial increase in drug entrapment as compared to the SLN formulations prepared with Tween 80 , because of the greater solubilizing properties of

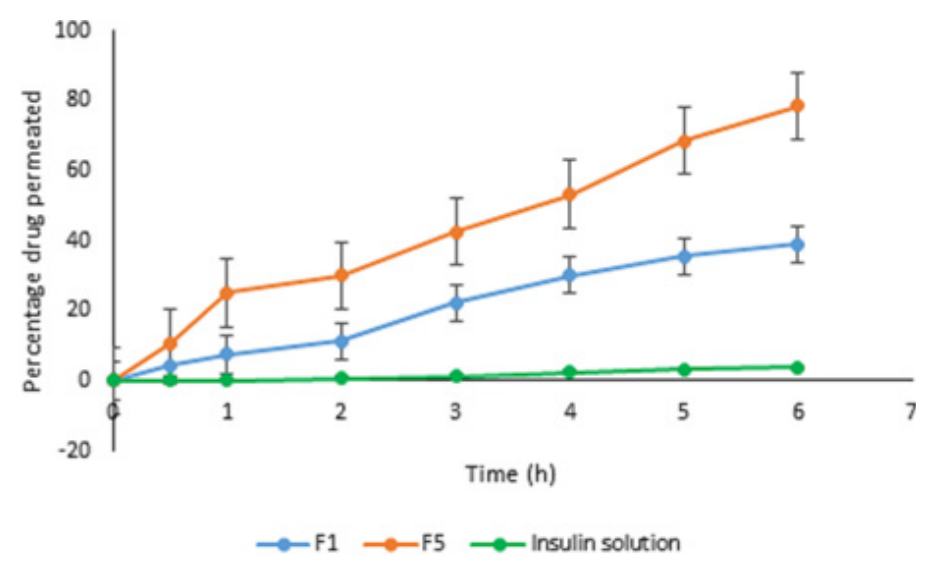

Figure 4: Percentage drug permeated profiles of insulin through intestinal mucosa of goat.

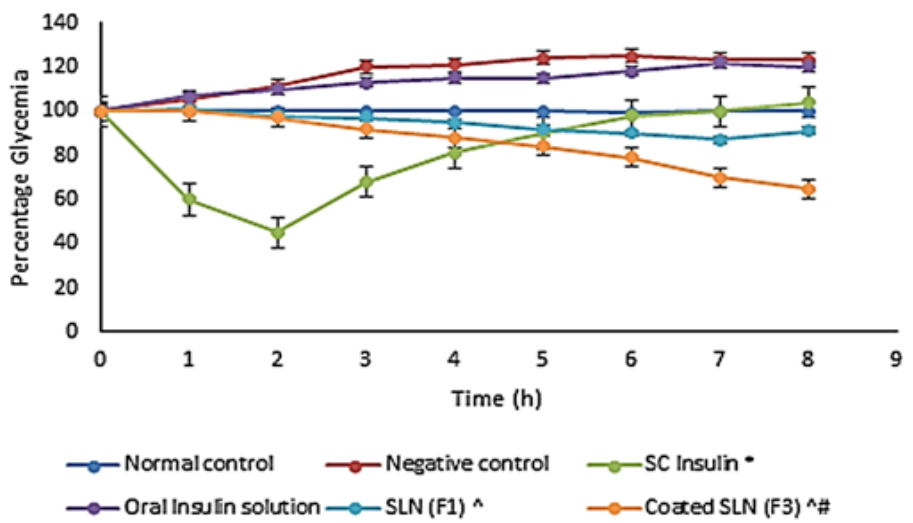

Figure 5: Percentage Glycemia in albino rats after administration of formulations.

SC - subcutaneous.

The values represent the mean \pm SEM for groups of 6 rats each. ${ }^{*} P<0.005$ when compared to negative control, normal control and oral insulin groups. $\wedge P<0.05$ when compared to oral insulin group. $\wedge \# P<0.05$ when compared to oral insulin and to F1 groups. 
the former. Between the two coated formulations, F3 and F4, the former was considered optimal in terms of not only AE and LE but also particle size and PDI and therefore was selected for further investigations.

The Caco-2 cell monolayer model is considered as the gold standard for intestinal permeation studies and therefore provide valuable information regarding intestinal absorption of drugs. Although the Caco-2 cell monolayer may not be an exact replica of the intestinal mucosa in terms of cell differentiation and presence of mucus secreting cells, it is however a reliable model of intestinal epithelial cells suitable for the study of drug transport through intestinal mucosa. ${ }^{33-35}$ The effect of the chitosan coating on insulin permeation was also considered by comparing with results from the uncoated form as well as from insulin solution. The results suggest that the chitosan coating makes a difference to the extent of insulin permeation through the monolayer. The percentage of insulin permeated from the solution $(2.3 \%)$ was almost negligible considering that a hydrophilic macromolecular drug would not be able to permeate through the lipid cell membrane by passive diffusion unless a paracellular pathway is available. However, the paracellular pathway for the transport of hydrophilic molecules does not exist in the Caco-2 cell monolayer model. ${ }^{36}$ Moreover, in vivo, the degradation of insulin by acids and enzymes would have produced a similar result. The SLN formulations produced a significant permeation $(P<0.005$ for $\mathrm{F} 3$ and $P<0.05$ for F1) through the monolayer when compared to the insulin solution. At the end of $6 \mathrm{~h}$, maximum percentage drug permeated from F3 was nearly 4 times that from F1 and 14 times that of insulin solution. Even the uncoated SLN, F1 produced a maximum permeation of $9.6 \%$ which was 4 times that from the insulin solution. This result could be attributed to the small size of the nanoparticles and the lipophilic characteristics imparted by the lipid matrix of the SLN to the hydrophilic drug which could enable the latter to be absorbed by the transcellular pathway. The chitosan coating on SLN of F3 produced the necessary muco-adhesion for the improved permeation or uptake into the cells as compared to the uncoated SLN. As there was a significant difference $(P<0.005)$ in the permeation from $\mathrm{F} 3$ as compared to $\mathrm{F} 1$, we concluded that the chitosan coating was largely responsible for the improved transport of the former through the Caco- 2 cell monolayer. ${ }^{34,35}$

The slow release of insulin from the SLNs in the permeation studies through goat intestinal mucosa, would be due to the combined mechanisms of water permeation through the lipid matrix followed by leaching out of the drug by diffusion through the swollen gelled chitosan layer as well as the slow erosion of the lipid matrix after the dissolution of the coating. There was a substantial increase in insulin permeation from the chitosan coated SLN as compared to the uncoated formulation or insulin solution. The fact that the insulin in solution form barely permeated the mucosa shows that a water soluble macromolecule would find it extremely difficult to permeate a lipoidal biological barrier. The superior permeation of the chitosan coated SLN could be attributed to its mucoadhesive properties which is responsible for localizing the particles on the mucosa. Chitosan also possesses permeability enhancing property by bringing about the temporary opening of tight junctions in the mucosal cell membrane. ${ }^{14,15}$

In vivo anti-diabetic studies in streptozotocin induced diabetic rats revealed that, in the positive control group that received SC insulin, there was an immediate fall in blood glucose levels. At the end of 8 $\mathrm{hr}$, a significant reduction in blood glucose levels was observed when compared to negative control, normal control or oral insulin groups $(p<0.005)$. The groups that received oral insulin solution produced blood glucose levels that were almost similar to that of the negative control groups in the first $2 \mathrm{hr}$ but declined slightly later. This difference was not statistically significant and could not be ascertained whether insulin absorption was the cause. On the other hand, the group that received uncoated SLN (F1) and coated SLN (F3) showed a gradual fall in blood glucose which could be attributed to the sustained release of insulin. The percentage glycemia produced at the end of $8 \mathrm{~h}$ in both groups was statistically significant as compared to the oral insulin $(p<0.05)$ group. The hypoglycemic levels produced by the groups that received F1 and F3 were similar initially, but after the third hour, the latter showed a significant $(p<0.05)$ fall in blood glucose levels below that of the group that received the former. At the end of $8 \mathrm{~h}$, the blood glucose levels achieved with F3 was almost similar to that produced by subcutaneous insulin in the third hour before the concentrations start climbing. Although the subcutaneous insulin was able to achieve normal glucose levels in the $2^{\text {nd }} \mathrm{hr}$, it was unable to maintain them beyond this time with the levels gradually coming to baseline levels at the end of $6 \mathrm{~h}$. The maximum fall in blood glucose levels of $87 \%$ was produced at $7 \mathrm{~h}$ by F1 whereas F3 continued to show further decreasing levels beyond $8 \mathrm{~h}$. Thus, the results indicated that the hypoglycemic effect was more sustained when SLN were coated with chitosan than otherwise. Moreover, it also means that SLN and especially chitosan coated SLN have the potential of improving the absorption of insulin through intestinal mucosa. Besides improving mucosal permeability, it is also possible that the chitosan coating may provide protection to insulin from degradation by intestinal enzymes and thus improve its bioavailability. ${ }^{37,38}$

\section{CONCLUSION}

In our study, we were successful in developing a promising oral form of insulin as chitosan coated solid lipid nanoparticles which could be a suitable and practical alternative to subcutaneous insulin without the disadvantages of the latter. This study shows that it is possible to design chitosan coated nanoparticles of insulin, with the properties necessary for better intestinal permeation and having controlled release properties. The oral administration of chitosan coated insulin SLN to diabetic rats resulted in significant hypoglycemic effect, which was more pronounced than that obtained after the administration of uncoated insulin-loaded SLN. Thus, chitosan coating was found to improve the stability and intestinal absorption properties of SLN containing insulin, which would contribute to the development of an optimized oral insulin formulation.

\section{ACKNOWLEDGEMENT}

The authors would like to express their gratitude to the NITTE (Deemed to be University), Mangalore for the use of the facilities and the funding necessary to carry out this project.

\section{CONFLICT OF INTEREST}

The authors declare no conflict of interest.

\section{ABBREVIATIONS}

SLN: Solid lipid nanoparticles; IDDM: Insulin dependent diabetes mellitus; NIDDM: Noninsulin dependent diabetes mellitus, GI: Gastro-intestinal; GMS: Glycerol monostearate; DLV: Laser Dropper velocimetry; PALS: Phase Analysis Light Scattering; SEM: Scanning electron microscopy; TEM: Transmission electron microscopy; PDI: Polydispersity index; AE: Association efficiency; LE: Loading efficiency; DMEM: Dulbecco's modified Eagle medium; CPCSEA: Committee for the Purpose of Control and Supervision of Experiments on Animals; ANOVA: Analysis of variance; SPSS: Statistical Package for the Social Sciences.

\section{REFERENCES}

1. Ahmad A, Othman L, Zain AZ, Chowdhury EH. Recent advances in insulin theraphy for diabetes. Int J Diabetes Clin Res. 2014;1(1):1-13.

2. Harsoliya MS, Pate VM, Modasiya M, Pathan JK, Chauhan A, Parihar M, et al. 
Recent advances and application of nanotechnology in diabetes. Int J Pharm Biol Sci Arch. 2012;3(2):255-61.

3. Mane K, Chaluvaraju K, Niranjan M, Zaranappa T, Manjuthej T. Review of insulin and its analogues in diabetes mellitus. J Basic Clin Pharm. 2012;3(2):283-93.

4. Sarmento B, Martins S, Ferreira D, Souto EB. Oral insulin delivery by means of solid lipid nanoparticles. Int J Nanomed. 2007;2(4):743-9.

5. Prusty AK, Sahu SK. Development and Evaluation of Insulin Incorporated Nanoparticles for Oral Administration. Int Scholarly Res Nanotech. 2013;5:5662.

6. Malathi S, Nandhakumar P, Pandiyan V, Webster TJ, Balasubramanian S. Nove PLGA-based nanoparticles for the oral delivery of insulin. Int $\mathrm{J}$ Nanomed. 2015;10(1):2207-18

7. Lin Y, Chen C, Liang H, Kulkarni A, Lee P, Chen C, et al. Novel nanoparticles for oral insulin delivery via the par cellular pathway. Nanotechnology. 2007;18(10):105102.

8. Rawat M, Singh D, Saraf S. Nanocarriers: Promising vehicle for bioactive drugs. Biol Pharm Bull. 2006;29(9):1790-8.

9. Muller RH, Maaen S, Weyhers H, Specht F, Lucks JS. Cytotoxicity of magnetiteloaded polylactide, polylactide/glycolide particles and solid lipid nanoparticles. Int J Pharm. 1996;138(1):85-94.

10. Muller RH, Ruhl D, Runge S. Biodegradation of solid lipid nanoparticles as a function of lipase incubation time. Int J Pharm. 1996;144(1):115-21.

11. Yadav P, Soni G, Mahor A, Alok S, Singh PP, Verma A. Solid lipid nanoparticles: An effective and promising drug delivery system: A Review. Int J Pharm Sci Res. 2014;5(4):1152-62.

12. Garcia-Fuentes M, Torres D, Alonso MJ. New surface-modified lipid nanoparticles as delivery vehicles for salmon calcitonin. Int J Pharm. ;296:122-32.

13. Sogias I, Williams A, Khutoryanskiy V. Why is Chitosan Mucoadhesive? Biomacromolecules. 2008;9(7):1837-42

14. Roy K, Mao H, Huang S, Leong K. Oral gene delivery with chitosan-DNA nanoparticles generates immunologic protection in a murine model of peanut allergy. Nature Medicine. 1999;5(4):387-91.

15. Borges O, Cordeiro-da-Silva A, Romeijn S, Amidi M, DeSousa A, Borchard $\mathrm{G}$, et al. Uptake studies in rat Peyer's patches, cytotoxicity and release studies of alginate coated chitosan nanoparticles for mucosal vaccination. J Control Release. 2006;114(3):348-58.

16. Zhang N, Ping $Q$, Huang G, Xu W, Cheng $Y$, Han $X$. Lectin modified solid lipid nanoparticles as carriers for oral administration of insulin. Int $\mathrm{J}$ Pharm. 2006;327(1-2):153-9.

17. Fonte P, Nogueira T, Gehm C, Ferreira D, Sarmento B. Chitosan-coated solid lipid nanoparticles enhance the oral absorption of insulin. Drug Deliv Transl Res. 2011;1(4):299-308

18. Miret S, Abrahamse L, Groene EM. Comparison of in vitromodels for the prediction of compound absorption across the human intestinal mucosa. J Biomol Screen. 2004;9(7):598-606.

19. Ranaldi G, Islam K, Sambuy Y. Epethelial cells in culture as a model for the intestinal transport of antimicrobial agents. Antimicrob Agents Chemother. 1992;36(7):1374-81.

20. Hafiz MMJ, Khuriah AH, Meor MMR, Liza S, Suzana MJ, Tommy BE. A simple and sensitive HPLC method for determination of insulin in rat plasma and its application in pharmacokinetic study. Int J Pharm Pharm Sci. 2013;5(2):133-7.

21. Chakraborti CK, Sahoo S, Behera PK. Effect of different polymers on in vitro and ex vivo permeability of Ofloxacin from its mucoadhesive suspensions. Saudi Pharm J. 2015;23(2):195-201.

22. Ragbetli C, Ceylan E. Effect of streptozotocin on biochemical parameters in rats. Asian J Chem. 2010;22(3):2375-8.

23. Toro-Equihua MD, Velasco-Rodriguez R, Lopez-Ascencio R, Vasquez C. Effect of an avocado oil-enhanced diet (Persea Americana) on sucrose-induced insulin resistance in wistar rats. J Food Drug Anal. 2016;24(2):350-7.

24. Zhang $H$, Wang $W$, Li H, Yi P, Zhang Z. Microspheres for the oral delivery of insulin: Preparation, evaluation and hypoglycaemic effect in streptozotocininduced diabetic rats. Drug Dev Ind Pharm. 2018;44(1):109-15.

25. Pan Y, Li Y, Zhao H, Zheng J, Xu H, Wei G, et al. Bioadhesive polysaccharide in protein delivery system: Chitosan nanoparticles improve the intestinal absorption of insulin in vivo. Int J Pharm. 2002;249(1-2):139-47.

26. Barua S, Mitragotri S. Challenges associated with penetration of nanoparticles across cell and tissue barrier: A review of current status and future prospects. Nano Today. 2014;9(2):223-43

27. Mudie DM, Amidon GL, Amidon GE. Physiological parameters for oral delivery and in vitro testing. Mol Pharm. 2010;7(5):1388-405.

28. Shekhawat PB, Varsha PB. Understanding peroral absorption: Regulatory aspects and contemporary approaches to tackling solubility and permeability hurdles. Acta Pharm Sin B. 2017;7(3):260-80.

29. Zirak MB, Pezeshki A. Effect of surfactant concentration on the particle size, stability and potential zeta of beta carotene nano lipid carrier. Int J Curr Microbiol App Sci. 2015;4(9):924-32

30. Shah R, Eldridge D, Polambo E, Harding I. Optimization and stability assessment of solid lipid nanoparticles using particle size and zeta potential. J Phys Sci. 2014;25(1):59-75.

31. Cortesi R. Production of lipospheres as carriers for bioactive compounds. Biomaterials. 2002;23(11):2283-94.

32. Kumar R, Yasir M, Saraf $S$, Gaur $P$, Kumar $Y$, Singh A. Glyceryl monostearate based nanoparticles of mefenamic acid: Fabrication and in vitro characterization. Drug Invention Today. 2013;5(3):246-50.

33. Gamboa J, Leong K. In vitro and in vivo models for the study of oral delivery of nanoparticles. Adv Drug Deliver Rev. 2013;65(6):800-10.

34. Yin L, Ding J, He C, Cui L, Tang C, Yin C. Drug permeability and mucoadhesion properties of thiolated trimethyl chitosan nanoparticles in oral insulin delivery. Biomaterials. 2009;30(29):5691-700.

35. Cano-Cebrian MJ, Zornoza T, Granero L, Polache A. Intestinal absorption enhancement via the paracellular route by fatty acids, chitosans and others: A target for drug delivery. Curr Drug Deliv. 2005;2(1):9-22.

36. Behrens I, Stenberg P, Artursson P, KisselT. Transport of lipophilic drug molecules in a new mucus-secreting cell culture model based on HT29-MTX cells. Pharm Res. 2001;18(8):1138-45.

37. Zhang N, Ping Q, Huang G, Xu W, Cheng Y, Han X. Lectin modified solid lipid nanoparticles as carriers for oral administration of insulin. Int $\mathrm{J}$ Pharm. 2006;327(1-2):153-9.

38. Zhang Z, Lv H, Zhou J. Novel solid lipid nanoparticles as carriers for oral administration of insulin. Pharmazie. 2009;64(9):574-8.

Article History: Submission Date : 17-10-2020; Revised Date : 09-01-2021; Acceptance Date : 01-02-2021

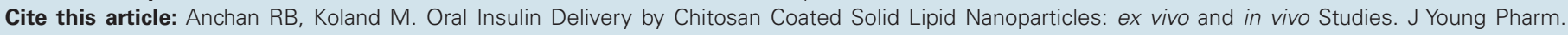
$2021 ; 13(1): 43-8$ 